\title{
A novel role for cardiac neural crest in heart development
}

\author{
Karen Waldo, Marzena Zdanowicz, Jarrett Burch, Donna H. Kumiski, Harriet A. Stadt, \\ Robert E. Godt, Tony L. Creazzo, and Margaret L. Kirby \\ Developmental Biology Program, Institute of Molecular Medicine and Genetics, Medical College of Georgia, \\ Augusta, Georgia 30912-2640, USA \\ Address correspondence to: Margaret L. Kirby, Developmental Biology Program, Institute of Molecular Medicine and Genetics, \\ Medical College of Georgia, Augusta, Georgia 30912-2640, USA. Phone: (706) 721-8774; Fax: (706) 721-8685; \\ E-mail: mkirby@mail.mcg.edu \\ Received for publication February 9, 1999, and accepted in revised form April 27, 1999.
}

\begin{abstract}
Ablation of premigratory cardiac neural crest results in defective development of the cardiac outflow tract. The purpose of the present study was to correlate the earliest functional and morphological changes in heart development after cardiac neural crest ablation. Within 24 hours after neural crest ablation, the external morphology of the hearts showed straight outflow limbs, tighter heart loops, and variable dilations. Incorporation of bromodeoxyuridine in myocytes, an indication of proliferation, was doubled after cardiac neural crest ablation. The myocardial calcium transients, which are a measure of excitation-contraction coupling, were depressed by $50 \%$ in both the inflow and outflow portions of the looped heart tube. The myocardial transients could be rescued by replacing the cardiac neural crest. The cardiac jelly produced by the myocardium was distributed in an uneven, rather than uniform, pattern. An extreme variability in external morphology could be attributed to the uneven distribution of cardiac jelly. In the absence of cardiac neural crest, the myocardium was characterized by somewhat disorganized myofibrils that may be a result of abnormally elevated proliferation. In contrast, endocardial development appeared normal, as evidenced by normal expression of fibrillin-2 protein (JB3 antigen) and normal formation of cushion mesenchyme and trabeculae. The signs of abnormal myocardial development coincident with normal endocardium suggest that the presence of cardiac neural crest cells is necessary for normal differentiation and function of the myocardium during early heart development. These results indicate a novel role for neural crest cells in myocardial maturation.
\end{abstract}

J. Clin. Invest. 103:1499-1507 (1999).

\section{Introduction}

The cardiac neural crest ablation model has been used extensively to study normal and abnormal cardiovascular development in the chick embryo. Cells of the cardiac neural crest are necessary for normal septation of the cardiac outflow tract and many of the derivatives of the caudal 3 pharyngeal arches, including the great arteries of the thorax, thyroid, thymus, and parathyroids (1). The cells of the cardiac neural crest (between the midotic placode and the third somite) leave the neural tube and migrate into the circumpharyngeal ridge, where they are poised to enter the caudal pharyngeal arches as they form (2). The pharyngeal arches are sequentially demarcated by the formation of pharyngeal pouches. Each arch becomes populated by cardiac neural crest cells, which condense around the nascent aortic arch artery $(3,4)$. After establishing residence in the caudal pharyngeal arches, a subpopulation of cardiac crest cells moves into the cardiac outflow tract to participate with other elements in outflow septation (5). Failure of outflow septation is a hallmark of cardiac neural crest ablation and is called persistent truncus arteriosus (PTA) (6-8). The common outflow vessel usually arises from the right ventricle and is always accompanied by abnormal patterning of the great arteries $(9,10)$.
A number of myocardial functional anomalies have been documented on days 11 and 15 of incubation after septation should have occurred $(11,12)$. Whereas it seems reasonable that myocardial functional changes occur after altered morphological development, an unresolved aspect of research in this model has been data showing that ventricular function is altered on day 3 of incubation, prior to the time that neural crest cells would reach the outflow tract, and well before outflow septation would begin in an intact embryo (13). The most severe alteration in ventricular function is decreased ejection fraction. In addition to changes in ventricular function, abnormal morphology of the heart loop in early neural crest-ablated embryos has been reported (7, 13-16). All of these data indicate that cardiac neural crest ablation affects early heart development.

During looping, the initially straight heart tube bends into a C-shape with the convex side pointed rightward $(17,18)$. This shape creates 2 limbs, an inflow (proximal) limb and an outflow (distal) limb. Subsequently, the proximal part of the inflow limb moves cranially toward the distal part of the outflow limb in a process called convergence (19), in which the inflow is repositioned from an initial caudal position to a cranial position and nestles behind the ventrally displaced outflow limb of the loop $(17,19)$. This convergence permits correct align- 
ment of the outflow septum with the atrioventricular and ventricular septa in the final stage of ventricular septation (1). Experimental studies have suggested that abnormal looping and convergence can cause malalignment of inflow and outflow segments of the hearts (7, 19-21). Cardiac neural crest ablation results in doubleoutlet right ventricle or rightward displacement of the PTA in $80 \%$ of the affected hearts after neural crest ablation $(1,7,9)$. Both are signs of incorrect convergence.

Although hemodynamic changes in the aortic arch arteries were expected to play a role in generating incorrect convergence (22), these changes were never consistently found by our group. (L. Leatherbury and D.M. Connuck, unpublished observations). If cardiac neural crest ablation results in hemodynamic alterations of the aortic arch arteries that subsequently cause abnormal ventricular function, then ablation of the arch arteries themselves should cause ventricular function to be altered, similar to neural crest ablation. Leatherbury and Connuck made lesions in the right pharyngeal wall prior to formation of pharyngeal arches 3 and 4, which caused a diminished number of right aortic arch arteries to form. The left aortic arch arteries appeared to compensate for resultant flow changes by increasing in size, and the embryos showed no evidence of decreased contractility (R. Hixon et al., manuscript submitted for publication). Similar compensation is seen in neural crest-ablated embryos (14), suggesting that it is unlikely that ventricular dysfunction in these embryos results from an increased afterload. Although a hemodynamic role in cardiac dysfunction after neural crest ablation cannot be ruled out, no evidence has been forthcoming to support this mechanism. Thus, we have begun to search for other factors that might lead to primary myocardial alterations.

This study is an initial attempt to find early abnormalities in cardiac development that might underlie abnormal convergence. Our findings indicate that the myocardial developmental program is perturbed for both structure and function within 24 hours after cardiac neural crest ablation. In contrast, endocardial development and production of cushion mesenchyme appear to be normal. Since cardiac neural crest cells would not have arrived in the heart or caudal pharyngeal arches at this time in an intact embryo, this finding suggests that neural crest cells must influence the developing myocardium at a distance and that their presence is essential for normal myocardial development well before the time they participate in outflow septation. This hypothesis is strengthened by experiments in which myocardial function is rescued when neural crest is replaced following extirpation.

\section{Methods}

Embryo preparation. Fertilized Arbor Acre chicken eggs (Seaboard Hatchery, Athens, Georgia, USA) were incubated at $37^{\circ} \mathrm{C}$ and $97 \%$ humidity in a forced-draft incubator. After 30 hours of incubation, windows were made in the eggs. The embryos were lightly stained with neutral red-impregnated agar and staged according to Hamburger and Hamilton (23). At stages 9 or 10, the cardiac neural folds were ablated bilaterally from the midotic placode to the posterior limit of somite 3 with a pulsed nitrogen/dye laser (VSL-377/DLM-110; Laser Science Inc., Newton, Massachusetts, USA; ref. 8). Sham-operated embryos were stained with neutral red only. Control eggs were unopened until the time of collection. The eggs of sham-operated and neural crest-ablated embryos were sealed with cellophane tape and reincubated.

A second set of embryos was produced by manual extirpation of the cardiac neural folds using electrolytically sharpened tungsten needles to produce "experimental" embryos. For "rescued" embryos, the folds were replaced into the ablation site just after they were detached. In both cases, the vitelline membrane was torn over the ablation site prior to the surgery. Shamoperated embryos were produced by tearing the vitelline membrane with no further surgery. The eggs were sealed with cellophane tape and reincubated.

On incubation days 2,3 , or 4 , the embryos were removed from the eggs and placed in a $1.8 \%$ buffered potassium chloride solution $(243 \mathrm{mM})$ until the hearts stopped beating in diastole. Each embryo was cleaned of extraembryonic membrane, staged, rinsed in normal saline, and placed in fixative. Two- and 3 -day embryos were staged by counting somites; day 4 embryos were staged by external morphological features (24). Fixation varied according to the procedure used on the embryos: $10 \%$ buffered formalin for morphometry, cold $4 \%$ paraformalde-

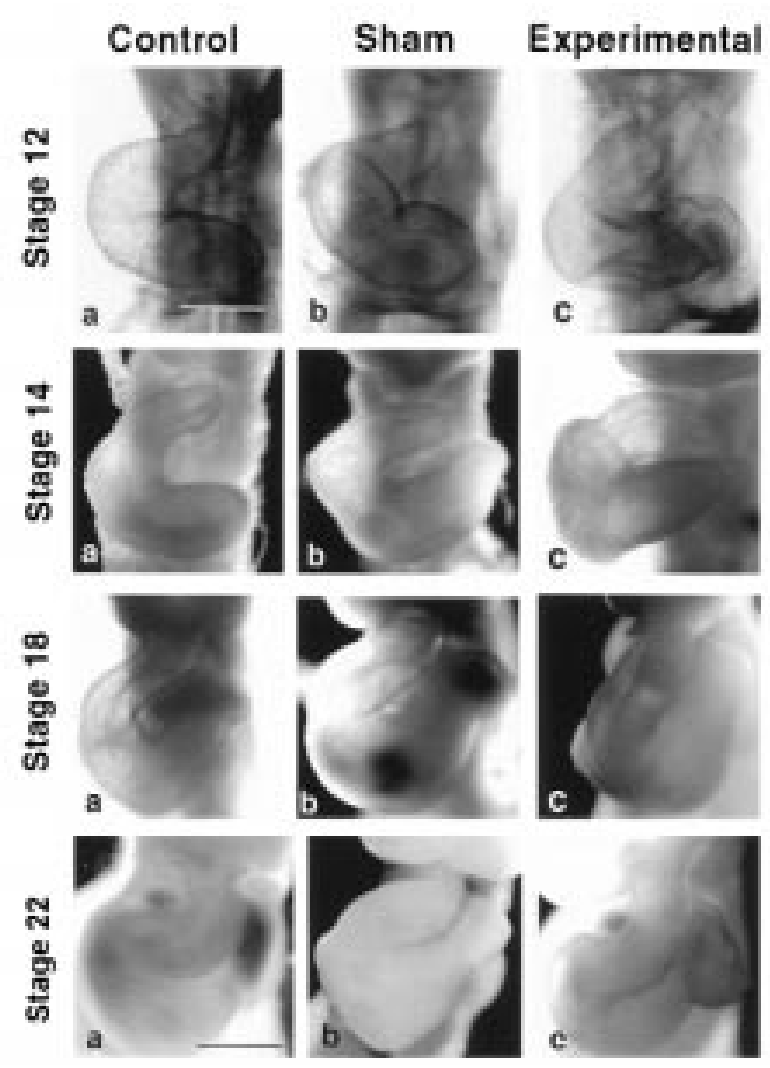

Figure 1

Cardiac development appears abnormal from 24 hours following cardiac neural crest ablation. Frontal views of hearts from stage 12, 14, 18, and 22 control (a), sham-operated (b), and neural crest-ablated (c) embryos. The normal space between the inflow and outflow limbs, as seen in the controls, is diminished or reduced in sham and experimental embryos. Straightened outflow limbs are first present in most of the stage 14 experimental embryos and can be seen in stage 18 experimental and sham hearts. At stage 18 , variable dilations of the heart tube occur in the experimental embryos. Note the distinctive bend of the conotruncus in the control heart at stage 22. Many of the experimental hearts have outflows with straighterthan-normal outflow limbs (stage 22, $\mathbf{b}$ and $\mathbf{c}$ ). Scale bars at stages 12-18: $0.33 \mathrm{~mm}$; scale bar at stage 22: $1 \mathrm{~mm}$ ). 

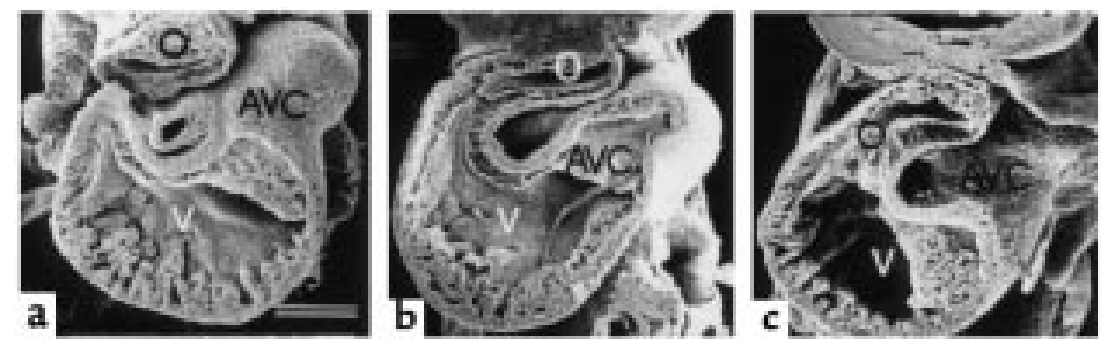

Figure 2

Trabeculation develops normally after neural crest ablation. Scanning electron micrographs of the heart of stage 19 chick embryos, sectioned in the frontal plane through the presumptive ventricles, demonstrate the trabeculation pattern of control (a), sham (b), and experimental (c) embryos. O, outflow tract; AVC, atrioventricular canal; V, presumptive ventricular area. Scale bar: $200 \mu \mathrm{m}$. The trabeculation pattern appears similar in control, sham, and experimental hearts. The number of trabeculae, their lengths and widths, and the intertrabecular spaces are comparable. Scale bar: $100 \mu \mathrm{m}$

hyde for scanning electron microscopy and immunohistochemistry, and cold $2 \%$ paraformaldehyde and HEPES buffer for rhodamine phalloidin.

Cardiac morphometry. An average of 10 embryos were collected for each control group, sham-operated (sham) group, and cardiac neural crest-ablated (experimental) group, at stages 12, 14,18 , and $22 / 23$. The collection and analysis was done twice with an intervening interval of approximately 1 year. A Zeiss stereomicroscope, equipped with a Zeiss camera, was used to photograph each embryo from the left side and from the front. All embryos of a particular stage were photographed, and prints were made at consistent magnifications. Morphometric measurements were made from tracings drawn on clear acetate that had been placed over each photograph. The measurements made for each stage were determined by the characteristics of the heart loop. Angle, distance, and area were measured using a digitizing tablet and SigmaScan software (version 3.90; Jandel Scientific, San Rafael, California, USA). Statistical analysis of these measurements was done using ANOVA, with appropriate post hoc testing for significantly different groups (SigmaStat 2.0; Jandel Scientific).

Myocardial proliferation. Bromodeoxyuridine (BrdU) incorporation was used as a measure of myocardial proliferation. BrdU was applied to both sham-operated and neural crest-ablated embryos at stage 15 .

Immunohistochemistry. After fixation, the embryos were dehydrated, cleared, and embedded in paraffin. The blocks were sectioned at $7 \mu \mathrm{m}$. The slides were blocked with $10 \%$ goat serum and incubated overnight in proliferating cell nuclear antigen (PCNA), anti-BrdU, JB3 supernatant (gift of Roger Markwald, Medical College of South Carolina, Charleston, South Carolina, USA), or $\alpha$-smooth muscle actin ( $\alpha$-SMA) at 1:1,200 (Sigma Chemical Co., St. Louis, Missouri, USA). After washing 1-3 hours in Tris and 5 minutes in Tris with $2 \%$ FBS, the slides were incubated in an FITC-labeled secondary antibody (Southern Biotechnology Associates, Birmingham, Alabama, USA) for 1 hour. After washing, the slides were stained with 4',6-diamidine-2'-phenylindole dihydrochloride (DAPI; Boehringer Mannheim Biochemicals, Indianapolis, Indiana, USA), viewed on an Olympus BX-40 epifluorescence microscope (Olympus barrier filters WABI and WU), and photographed by double exposure on TMAX-400 Kodak film. Somites in each section served as a positive control for $\alpha$-SMA, and atrioventricular cushions served as a positive control for JB3. Negative controls were made by omitting the primary antibodies from selected sections.

Scanning electron microscopy. To compare the development of trabeculation in the different groups, control $(n=2)$, shamoperated $(n=2)$, and neural crest-ablated embryos $(n=3)$ were collected at stage 19 . After fixation, the embryos were transferred to $20 \%$ sucrose/PBS at $4{ }^{\circ} \mathrm{C}$ overnight and infiltrated with
$7.5 \%$ gelatin (Sigma Chemical Co.) in $15 \%$ sucrose for $1-5$ hours. The embryos were positioned in embedding molds filled with gelatin infiltration solution that was allowed to solidify. The blocks were frozen completely and sectioned on the cryostat until the desired depth within the heart was reached. The blocks were placed in phosphate buffer at $37^{\circ} \mathrm{C}$ until the gelatin melted, and the remaining embryos were rinsed in $0.2 \mathrm{M}$ cacodylate buffer, osmicated at room temperature for $30 \mathrm{~min}$ utes (osmium tetroxide diluted 1:3 with $0.2 \mathrm{M}$ cacodylate buffer), dehydrated in alcohol, and stored in absolute methanol at $4^{\circ} \mathrm{C}$. The embryos were processed in a Tousimis Sandri-790 critical point drier using liquid carbon dioxide; they were then mounted on stubs, coated with a 15 - to 20 -nm gold layer (Hummer gold sputterer; Technics, Inc., Alexandria, Virginia, USA), and examined by scanning electron microscope (XL-30 FEG scanning electron microscope, Philips Electronics, Eindhoven, the Netherlands).

Confocal microscopy. The fixed embryos were stained with rhodamine phalloidin using the procedure previously described by Price et al. (25). The embryos were washed in PBS with $0.02 \%$ azide (PBS-A) and $0.1 \%$ Triton X-100 for 1 hour, and for 30 minutes in PBS-A alone. Subsequently, they were placed in $1,500 \mathrm{U} / \mathrm{mL}$ hyaluronidase (Sigma Chemical Co.) for $45 \mathrm{~min}$ utes, $0.01 \mathrm{M}$ glycine for 1 hour, and 1\% BSA for 1 hour, before an overnight incubation in rhodamine phalloidin (Molecular Probes Inc., Eugene, Oregon, USA). After washing in PBS-A, the embryos were viewed on an MRC-1000 confocal microscope (Bio-Rad Laboratories Inc., Hercules, California, USA).

Intracellular myocardial calcium transients. Heart tubes were dissected at stages 14 or 18, washed in Ringer's solution, and the primitive atrium and outflow portions of the tubes were removed. The presumptive ventricular tissue was placed in $3 \mu \mathrm{M}$ fura-2/AM for 3 minutes at $37^{\circ} \mathrm{C}$ in an orbital shaker water bath as described previously (26). Excess dye was washed off with additional Ringer's solution, and intracellular de-esterification of fura-2/AM was allowed to proceed at room temperature for 25-30 minutes.

\section{Table 1}

Mean $( \pm$ SEM) number of cells per 50 counted that are labeled by BrdU in myocardium from sham-operated (Sham) and neural crest-ablated (Exp) embryos

\begin{tabular}{lcccc} 
& Outflow & $\begin{array}{c}\text { Inner } \\
\text { curvature }\end{array}$ & $\begin{array}{c}\text { Outer } \\
\text { curvature }\end{array}$ & Inflow \\
Sham & $12.5 \pm 1.4$ & $7.8 \pm 9.0$ & $11.8 \pm 1.3$ & $11.0 \pm 1.4$ \\
Exp & $24.0 \pm 3.4$ & $14.8 \pm 2.1$ & $26.0 \pm 1.6$ & $24.8 \pm 2.2$ \\
$P$ value & 0.009 & 0.013 & 0.001 & 0.002 \\
\hline
\end{tabular}

A minimum of 4 hearts were used for each group. 


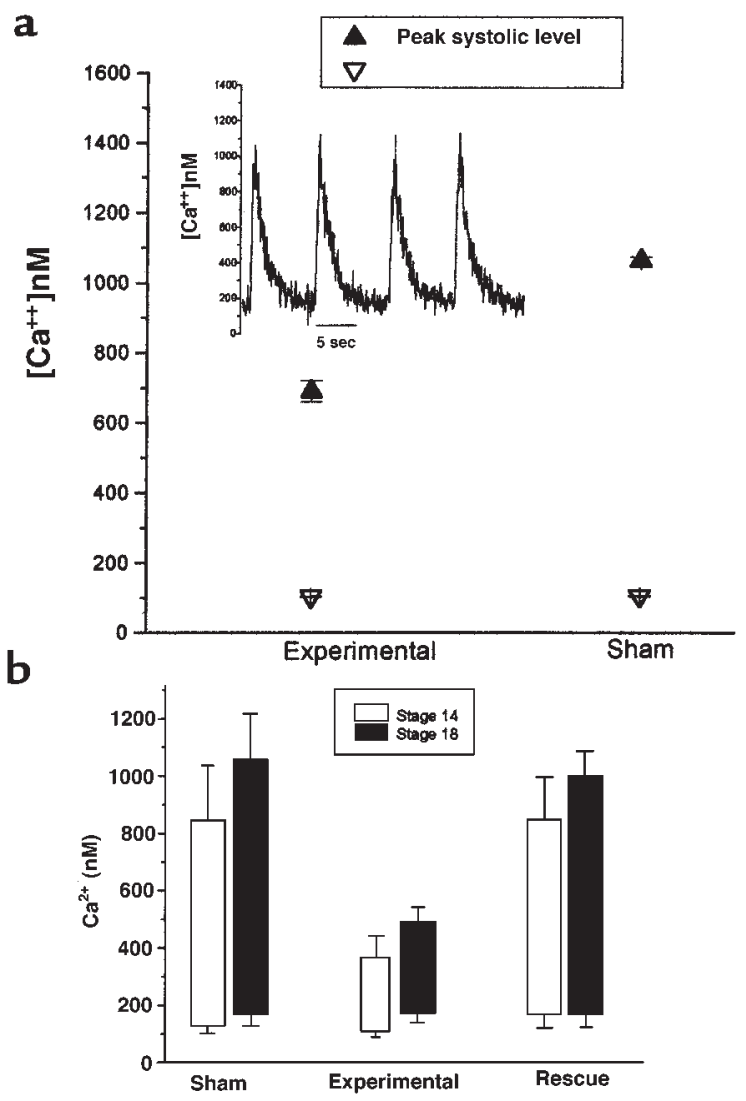

Figure 3

Intracellular $\mathrm{Ca}^{2+}$ transients in developing chick myocardium. (a) Average diastolic and peak systolic $\mathrm{Ca}^{2+}$ levels in presumptive ventricle of experimental and sham-operated (Sham) hearts at stage 18. The peak systolic $\mathrm{Ca}^{2+}$ level was significantly less in the experimental ventricles $(P<$ 0.001 , Student's $t$ test). $\mathrm{Ca}^{2+}$ transients were elicited by field stimulation at $0.3 \mathrm{~Hz}$. Inset shows an example of transients from presumptive ventricle of a sham embryo. Bars indicate SE. (b) Bar graph illustrating the intracellular $\mathrm{Ca}^{2+}$ transients elicited by field stimulation at $0.2 \mathrm{~Hz}$ at stages 14 and 18 in sham-operated, experimental, and rescued embryos. The top of each bar shows the average peak systolic $\mathrm{Ca}^{2+}$, and the bottom of each bar shows the average diastolic $\mathrm{Ca}^{2+}$. The peak systolic $\mathrm{Ca}^{2+}$ in experimental embryos at both stages is significantly depressed. Replacing the neural folds after extirpation rescued the normal transient value at both stages. A total of 6-8 embryos were examined in each case, and the groups were tested by ANOVA followed by either Student's $t$ test or Mann-Whitney rank sum test $(P<0.05)$.

This process results in a cytoplasmic concentration of fura- 2 of 25-30 $\mu \mathrm{M}(26,27)$. For measurement of the calcium transients, the fura-loaded ventricle was placed on the bottom of a $1-\mathrm{mL}$ coverslip chamber on the stage of an Olympus IX-70 inverted microscope equipped with a $\times 40$ oil-immersion objective (Olympus $\mathrm{UAPO} / 340$, numerical aperture $=1.35$; Olympus America Inc., Lake Success, New York, USA). The ventricle was electrically stimulated with fine platinum electrodes. For each experiment, data were collected from the central regions of the isolated ventricle. Motion artifacts were not detectable under these conditions. The intracellular calcium transients were estimated from the fluorescence output at $510 \mathrm{~nm}$, excited sequentially at 340 and $380 \mathrm{~nm}$ using a PTI DeltaScan-1 microspectrofluorometer that was optically coupled to the inverted microscope. Conversion of the fluorescence output to intracellular calcium levels is described by Brotto and Creazzo (26).

In a second series of experiments, the descending and ascend- ing limbs of the cardiac loop were isolated separately by removing the loop from the pharyngeal region and bisectioning it at the primary fold.

\section{Results}

Alterations in heart looping. Extreme variability was the overriding characteristic in external heart morphology in the experimental embryos. While the experimental embryos were almost always obvious because of the irregularities in heart looping, the morphometric measurements showed no significant differences. A majority of the experimental hearts showed variations in heart loop morphology, including tighter looping, straighterthan-normal and ventrally displaced outflow limbs, and the presence of variable dilations along the heart tube. Slightly older embryos displayed an unpredictable combination of abnormal and normal characteristics, such that no two hearts looked the same (Figure 1). These characteristics persisted through stage 23 (approximately incubation day 4).

Even with these macroscopic changes in external heart morphology, the cardiac trabeculae that developed during the same period appeared in sham and experimental heart tubes at the normal time and were similar in length, width, and intertrabecular spaces (Figure 2).

Abnormal myocardial development. Both functional and morphological parameters of myocardial development were measured in an attempt to obtain a complete profile of the earliest changes in heart development. Initial studies using PCNA staining suggested that the number of proliferating myocardial cells was elevated in the neural crest-ablated embryos. The extreme variability of this technique led us to use BrdU incorporation as a more stable indication of proliferation. Cells were counted at 4 separate sites in the myocardium of stage 15 embryos, and elevated incorporation of BrdU was found at each of the sites in the neural crest-ablated embryos (Table 1).

We measured intracellular calcium transients in the presumptive ventricular region of the cardiac loop as an indicator of the status of myocardial excitation-contraction coupling. While the diastolic calcium was similar in sham and experimental heart tubes, there was a dramatic reduction in the peak of the systolic calcium in experimental myocardium to approximately $600 \mathrm{nM}$, compared with about $1,100 \mathrm{nM}$ in the sham myocardium at stage 18 (Figure 3a). To determine whether this deficit could be specifically attributed to neural crest removal, in some embryos the neural crest was replaced into the lesion site just after its removal. These embryos showed normal myocardial calcium transients as compared with sham and experimental myocardium (Figure 3 , $a$ and $b$ ). Because the cardiac neural crest is in the pharyngeal region near the entrance of the outflow tract, we reexamined calcium transients in inflow versus outflow limbs of the cardiac loop (Table 2). The calcium transient was the same in the inflow and outflow portions of the tube at both stages 14 and 18, and both sites showed equivalent decreases in the calcium transients in neural crest-ablated embryos, as compared with sham-operated embryos (Table 2).

Histological analysis of the heart tubes at stages 
Table 2

Mean ( $\mathrm{nM} \pm \mathrm{SEM}$ ) of total systolic calcium transient measured in outflow and inflow limbs of hearts at stages 14 and 18

\begin{tabular}{lcc}
\hline & Sham & Experimental \\
Outflow & & \\
Stage 14 & $890.6 \pm 56.3$ & $458.3 \pm 66.5$ \\
Stage 18 & $846.9 \pm 21.4$ & $455.0 \pm 12.3$ \\
& & \\
Inflow & & \\
Stage 14 & $843.8 \pm 24.0$ & $425.0 \pm 76.9$ \\
Stage 18 & $862.5 \pm 26.3$ & $458.2 \pm 15.4$
\end{tabular}

Eight shams were measured at each stage, 6 experimental embryos at stage 14, and 5 at stage 18 . The outflow and inflow values were paired. The differences between sham and experimental outflow and inflow limbs are significantly different $(P=$ $0.05)$. The outflow and inflow limbs are not different in either the sham or in the experimental hearts.

14-16 showed that the sleeve of cardiac jelly, which has a characteristically uniform thickness through the length of the tube in sham-operated embryos, was distributed in a variable pattern in experimental hearts (Figure 4). The variability in the experimental hearts probably contributed to the random nature of the dilations in the loop and our inability to measure consistent changes in these obviously abnormal hearts. Because cardiac jelly is produced by the myocardium (28), alterations in its production could be an indicator of myocardial developmental status.

Having found these signs of abnormal myocardial development in neural crest-ablated embryos, we sought other markers of myocardial alterations. $\alpha$-SMA is one of the first contractile proteins expressed by the myocardium. In the chicken embryo, it is first expressed between stages 7 and 9; by stage 12, its expression becomes diminished and is replaced by $\alpha$-cardiac actin, except in the outflow tract (29-31). To see if the temporal pattern of expression of $\alpha$-SMA was changed in experimental embryos, serially sectioned hearts from stage 18 (incubation day 3 ) embryos were stained with an antibody against $\alpha$-SMA. The expression pattern was identical in all groups. Only the walls of the distal outflow tract had strong levels of $\alpha$-SMA expression (Figure 5), with weaker expression in the left wall of the atrioventricular canal and the left side of the left atrium, and minimal expression elsewhere in the heart.

F-actin is a component of the I-band and is therefore a marker of myofibrillogenesis (25). Whole-mount embryos were stained with rhodamine phalloidin and viewed by confocal microscopy to demonstrate F-actin (32). The staining pattern appeared different in control, sham, and experimental hearts (Figure 6). At higher magnification, it was obvious that the myofibrillar pattern in the sham and control hearts was better organized, with a higher degree of myofibrillar bundling and alignment (Figure 6).

Normal endocardial development. We used mesenchymal seeding of the atrioventricular endocardial cushions, which occurs at stages $15 / 16$, as an independent marker of endocardial development. JB3 antibody recognizes fibrillin-2, which is initially expressed throughout the endocardium but during looping (stages 15/16) becomes restricted to the atrioventricular and outflow tract cushions (33). At stage 14, the expression of fibrillin-2 was similar in all the groups (data not shown). There were no mesenchymal cells in the extracellular matrix of the cardiac jelly and no cells undergoing epithelial-mesenchymal transformation in the cushion areas. At stage 15, the epithelial-mesenchymal transformation had begun, and all embryos, regardless of treatment, had similar numbers of mesenchymal cells in the cardiac jelly of the atrioventricular cushions (Figure 5). This suggests that specific aspects of myocardial development are altered, whereas endocardial development is normal.

\section{Discussion}

Beginning just 24 hours after cardiac neural crest ablation, myocardial development is abnormal (Figure 7). Shortly preceding, or coincident with, the time when cardiac neural crest would normally be populating the caudal pharyngeal arches, the myocardium already shows abnormal proliferation, abnormal intracellular calcium transients, uneven production of cardiac jelly, and disarrayed myofibrils. A combination of these factors is likely to contribute to the macroscopically abnormal appearance of the cardiac loop. Even with these myocardial changes, the endocardium appears to be developing normally.

Myofibrillogenesis and electrical activity begin in the myocardium about stage $7.5-8$ in the chick $(31,34,35)$, with striated myofibrils appearing in scattered cardiomyocytes at about stage 10 , when the first weak contractions can be seen $(29,36-38)$. Anterograde circulation of blood begins at stage 14 (39), coincident with the time at which the first myocardial abnormalities can be seen in the absence of cardiac neural crest. The course of myocardial development appears to be altered beginning from the time at which neural crest cells would migrate to the circumpharyngeal ridge and populate pharyngeal arch 3 . This period of time is several days before neural crest cells would enter the outflow tract and have the opportunity to contact the myocardium of the outflow pole directly, suggesting that during early migration, neural crest cells affect myocardial development from a dis-
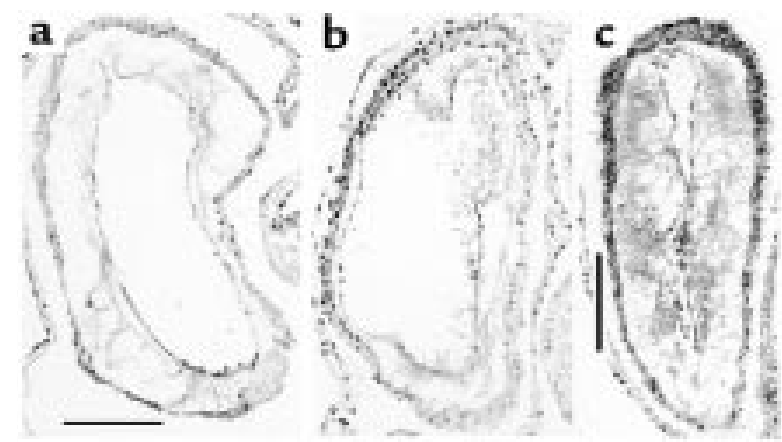

\section{Figure 4}

Accretion of cardiac jelly is abnormal after neural crest ablation. Transverse sections of the ascending limb of the cardiac loop at stage 14, comparing the uniform thickness of the cardiac jelly in sham-operated hearts (a) with the extreme variability in cardiac jelly disposition in hearts from embryos lacking cardiac neural crest (b and $\mathbf{c})$. The sections are stained for PCNA, and the increased proliferation in the myocardium in the experimental hearts is obvious. 


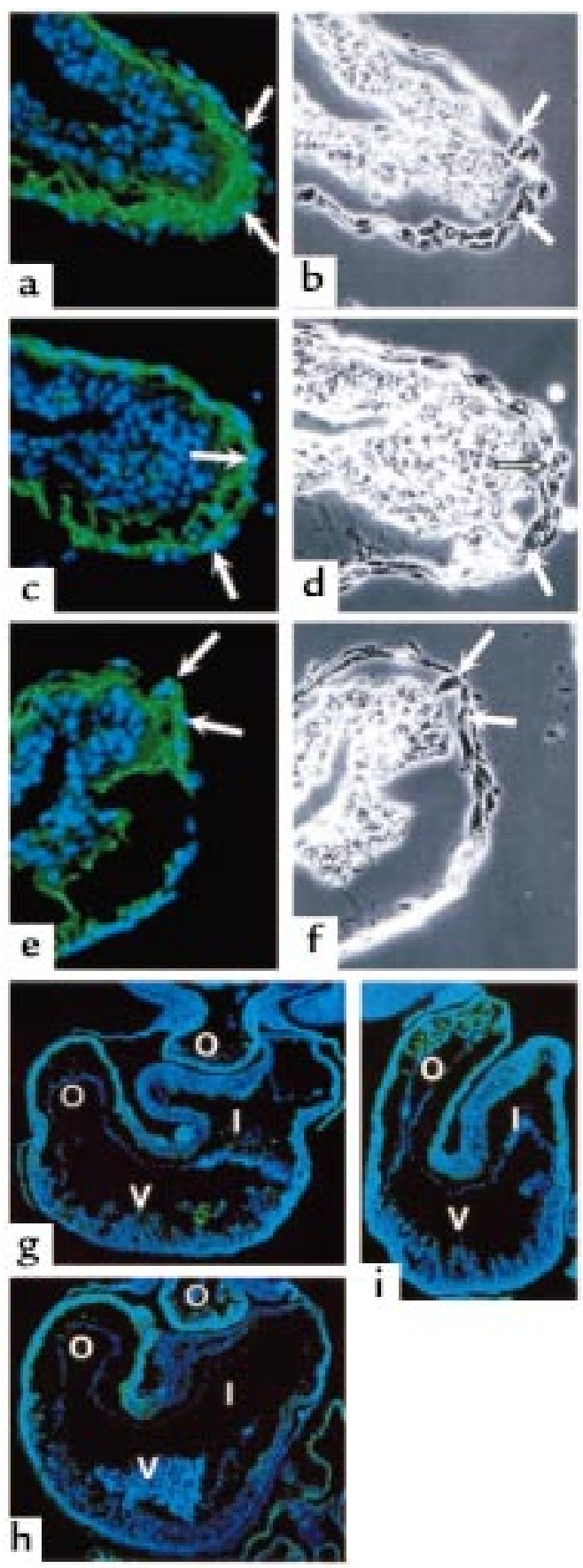

\section{Figure 5}

Formation of cushion mesenchyme and expression of $\alpha$-SMA are normal after neural crest ablation. Expression of JB3 in the atrioventricular canal of stage 15 control ( $\mathbf{a}$ and $\mathbf{b}$ ), sham ( $\mathbf{c}$ and $\mathbf{d}$ ), and experimental (e and f) hearts, and expression of $\alpha$-SMA in the heart loops of stage $18 \mathrm{control}$ (g), sham (h), and experimental (i) hearts. (a, c, and e) The endocardium and cardiac jelly of the atrioventricular canal are positive for the antibody JB3. All nuclei are labeled with DAPI (blue). White arrows point to endocardial cells that are undergoing epithelial-to-mesenchymal transition to seed the cardiac jelly with mesenchyme. (b, $\mathbf{d}$, and $\mathbf{f}$ ) Phase-contrast images of the sections seen in $\mathbf{a}, \mathbf{c}$, and $\mathbf{e}$. (g-i) Positive labeling for $\alpha$-SMA (light blue-green) is expressed in the outflow tracts $(O)$ of control, sham, and experimental hearts and, to a lesser degree, in the left wall of the atrioventricular canal and atrium. Although the ventricular wall appears somewhat positive for $\alpha$-SMA in the experimental heart (i), the ventricular walls in 2 other experimental hearts were not positive. I, inflow tract; $V$, presumptive ventricles. tance. Formation of the trabeculae involves both endocardium and myocardium, but recent data indicate that a neuregulin (huregulin) signal to initiate trabeculation emanates from the endocardium and may be additional evidence that the endocardium is developing normally in the absence of cardiac neural crest (40).

Altered myocardial development. F-actin, which labels Ibands of the contractile apparatus, was used to evaluate the status of cardiac myofibrillogenesis at stage 14 (34). It showed that hearts from embryos without cardiac neural crest have disordered myofibrils, as compared with shamoperated controls. Even so, the amount and distribution of $\alpha$-actin appeared normal, suggesting that expression of the contractile proteins may be normal. Unlike skeletal muscle, which completes proliferation prior to differentiation of the contractile apparatus, the myocardium must differentiate its contractile apparatus in order to function while growth occurs. This differentiation is possible because the contractile apparatus becomes mildly disorganized during myocardial cell division (41-43). The mild degree of myocardial disarray in neural crest-ablated embryos may be a direct result of elevated myocardial proliferation in the myocardium of these embryos.

The intracellular calcium transient is required for activation of the contractile apparatus and is central to the excitation-contraction coupling process (44). Automatic contractions occur in the myocardium of neural crest-ablated embryos, but in the presence of greatly reduced peak systolic intracellular calcium. Because myocardial cells at this age may not have much sarcoplasmic reticulum, the calcium transient is largely based on influx of extracellular calcium through sarcolemmal T- and L-type calcium channels (45). Creazzo et al. (46) have shown reduced L-type calcium current after neural crest ablation as early as 3 days of incubation. Thus, the reduced calcium transient reported here is likely due to reduced L-type calcium current (45). This reduction probably accounts for most of the observed decrease in contractility observed by Leatherbury and Connuck, although some could be the result of delayed myofibrillogenesis. Decreased calcium transients and Ltype calcium current have also been seen after neural crest ablation in myocardium at incubation days 11 and $15(11,27,47)$.

In addition to depressed excitation-contraction coupling and myofibrillar disarray, we have shown abnormal accretion of cardiac jelly in hearts from neural crest-ablated embryos. Cardiac jelly is produced by the myocardium. Taken together, these features suggest that myocardial development is altered, perhaps in a nonuniform manner. Both excitation-contraction coupling and myocardial contraction remain abnormal throughout development $(12,27,45,48,49)$, suggesting that the period of early myocardial function while anterograde circulation is first established defines a critical window of myocardial differentiation. Thus, we believe that disruption of normal neural crest function leads to a series of alterations in the myocardial developmental program that underlie the abnormal myocardial characteristics seen in hearts after neural crest ablation. These data suggest the presence of an unidentified factor produced or suppressed by the neural crest 

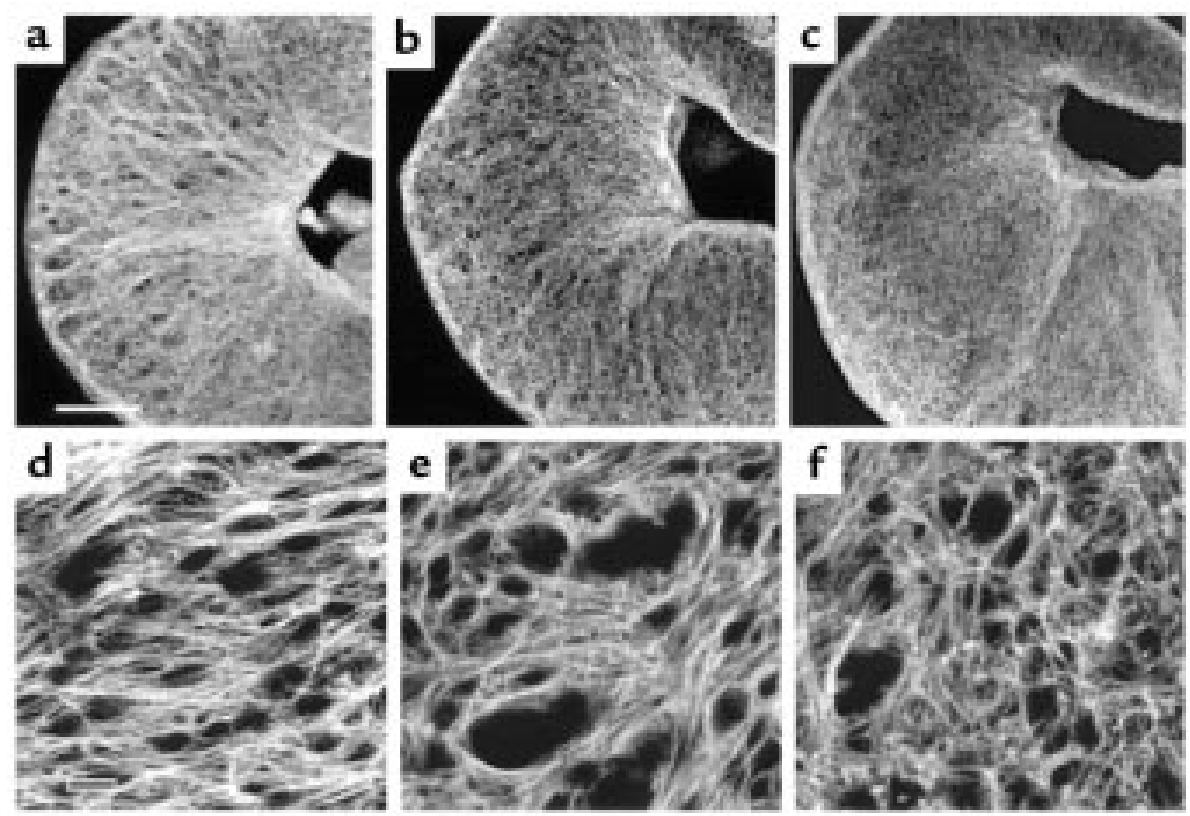

\section{Figure 6}

Cardiac myofibrils are disorganized after cardiac neural crest ablation. Rhodamine-phalloidin images of stage 14 chick heart obtained by confocal microscopy. Whole-mount hearts from control ( $\mathbf{a}$ and $\mathbf{d}$ ), sham-operated ( $\mathbf{b}$ and $\mathbf{e}$ ), and neural crest-ablated ( $\mathbf{c}$ and $\mathbf{f}$ ) embryos showing low- and high-magnification views of the same hearts. Slight dilation can be seen in this experimental heart. At low magnification, the staining pattern in the experimental heart is more diffuse than that in the control and sham hearts. Higher magnification shows that the myofibrillar pattern in the control and sham hearts appears better organized with more striations. Scale bar in a-d: $100 \mu \mathrm{m}$; scale bar in d-f: $10 \mu \mathrm{m}$.

that is critical to this early period of myocardial development. This interaction of cardiac neural crest with developing myocardium occurs much earlier and independently of neural crest participation in septation of the cardiac outflow tract.

The potential sources include a factor released by neural crest cells themselves that is required for normal myocardial development. In this case, removal of the cardiac neural crest would make this factor unavailable. In support of this mechanism, recent findings from 2 separate studies of $R X R a^{-/-}$mice (50,
51) support the concept that paracrine factors from extracardiac cells such as the neural crest may be critical in ventricular muscle growth and development. $\mathrm{RXR}^{-/-}$mice display cardiac ventricular and neural crest-related defects, supporting the model proposed by this study in chicks. Another possibility is that neural crest cells interact with another cell population to induce a factor that is needed for normal myocardial development. Alternatively, the neural crest cells could suppress production of a factor from a third population of cells.
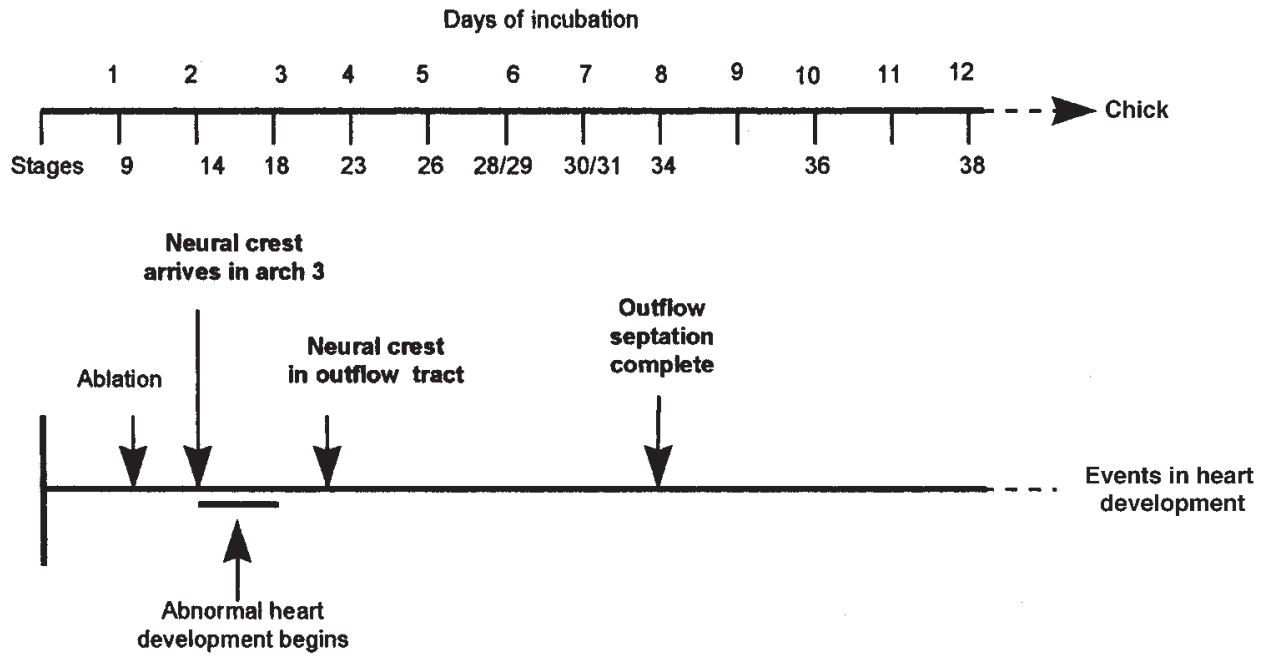

Figure 7

Time line summarizing the relationship of events in cardiac neural crest migration and cardiac development. Myocardial function is compromised well before the time when neural crest migrates into the outflow tract. 
The visceral endoderm, recently identified as the head organizer (52), has been recognized for its role in heart induction for many years (53-57). The heart tube lies directly ventral to the pharyngeal endoderm, which continues to release factors long after stable myocyte phenotypes are established (ref. 57; Farrell and Kirby, unpublished observations). Farrell et al. (manuscript submitted for publication) have recently cocultured endoderm and myocardium to show the same changes in myocardial proliferation and calcium transient; bFGF-2 induces the same changes. The effect of both endoderm and FGF-2 is blocked by adding a polyclonal anti-FGF neutralizing antibody to the cultures, suggesting that the endoderm releases an FGF-like factor during the time that neural crest cells would be populating the caudal pharyngeal arches $(4,58)$. This is at the same time that the initial changes can be documented in myocardial development in neural crest-ablated embryos. We propose the hypothesis that the myocardial abnormalities documented in the present study are caused by a deleterious influence of the pharyngeal endoderm on the myocardium due to an unnaturally prolonged period of release of an FGF-like factor (58).

Structural consequences of abnormal myocardial development. It is reasonable to suspect that alterations in myocardial development may lead to abnormal cardiac morphogenesis. Aberrant heart looping has the potential to alter alignment of the cardiac septa, resulting in major heart defects. In the neural crest ablation model, the configuration of the loop in early stages of heart development is predictive of abnormal convergence and/or wedging of the outflow tract between the atrioventricular valves in later development (7).

The early changes in myocardial function and morphology after neural crest ablation appear to lay the groundwork for abnormalities in cardiac looping and convergence. Because we do not know how well the heart can realign itself to compensate for slight malalignments of the inflow and outflow limbs, it is not possible to say with complete confidence that they result in malignment defects of the postseptation heart. However, because these malalignments are correlated with the earlier changes noted here, we believe that early myocardial abnormalities may be a factor in cardiac malalignment defects.

Functional attenuation of chick HIRA, a DiGeorge candidate gene, in the cardiac neural crest causes an increased incidence of PTA, a structural defect associated with cardiac neural crest ablation, but does not cause the other signs of neural crest ablation, namely, ventricular dysfunction, arch artery dysmorphogenesis, or outflow tract malalignment (58). The absence of ventricular functional abnormalities or outflow malalignment in these hearts suggests that attenuation of chick HIRA expression in the cardiac neural crest does not affect the role played by neural crest in early myocardial development. Furthermore, the fact that outflow tract alignment tends to be normal - in this case, in the absence of outflow tract septation - supports the hypothesis that early myocardial development plays a major role in outflow tract alignment (M.J. Farrell et al., manuscript submitted for publication)(58).

\section{Acknowledgments}

We thank Vicente Evangelista for help preparing the embryos and for photography; Penny Roon for her expert technical assistance and support with the scanning electron microscopy; Michael Farrell for valuable discussions of the experimental paradigm and critical reading of the manuscript; and Roger Markwald for the gift of the JB3 antibody. This work was supported by US Public Health Service grants HL-36059, HL51533, and HD-17063.

1. Kirby, M.L., and Waldo, K.L. 1995. Neural crest and cardiovascular patterning. Circ. Res. 77:211-215.

2. Kuratani, S.C., and Kirby, M.L. 1991. Initial migration and distribution of the cardiac neural crest in the avian embryo: an introduction to the concept of the circumpharyngeal crest. Am. J. Anat. 191:215-227.

3. Le Lièvre, C.S., and Le Douarin, N.M. 1975. Mesenchymal derivatives of the neural crest. Analysis of chimaeric quail and chick embryos. J. Embryol. Exp. Morphol. 34:125-154.

4. Waldo, K.L., Kumiski, D., and Kirby, M.L. 1996. Cardiac neural crest is essential for the persistence rather than the formation of an arch artery. Dev. Dyn. 205:281-292.

5. Kirby, M.L., Gale, T.F., and Stewart, D.E. 1983. Neural crest cells contribute to aorticopulmonary septation. Science. 220:1059-1061.

6. Kirby, M.L., Turnage, K.L., and Hays, B.M. 1985. Characterization of conotruncal malformations following ablation of "cardiac" neural crest. Anat. Rec. 213:87-93.

7. Tomita, H., Connuck, D.M., Leatherbury, L., and Kirby, M.L. 1991. Relation of early hemodynamic changes to final cardiac phenotype and survival after neural crest ablation in chick embryos. Circulation. 84:1289-1295.

8. Kirby, M.L., Kumiski, D.H., Myers, T., Cerjan, C., and Mishima, N. 1993. Backtransplantation of chick cardiac neural crest cells cultured in LIF rescues heart development. Dev. Dyn. 198:296-311.

9. Nishibatake, M., Kirby, M.L., and van Mierop, L.H. 1987. Pathogenesis of persistent truncus arteriosus and dextroposed aorta in the chick embryo after neural crest ablation. Circulation. 75:255-264.

10. Kirby, M.L., and Waldo, K.L. 1990. Role of the neural crest in congenital heart disease. Circulation. 82:332-340.

11. Aiba, S., and Creazzo, T.L. 1992. Calcium currents in hearts with persistent truncus arteriosus. Am. J. Physiol. 262:H1182-H1190.

12. Godt, R.E., Fogaça, R.T.H., and Nosek, T.M. 1998. Alterations of myocardial contraction associated with a structural heart defect in embryonic chicks. Adv. Exp. Med. Biol. In press.

13. Leatherbury, L., Gauldin, H.E., Waldo, K.L., and Kirby, M.L. 1990. Microcinephotography of the heart in neural crest-ablated chick embryos. Circulation. 81:1047-1057.

14. Bockman, D.E., Redmond, M.E., Waldo, K., Davis, H., and Kirby, M.L. 1987. Effect of neural crest ablation on development of the heart and arch arteries in the chick. Am.J. Anat. 180:332-341.

15. Bockman, D.E., Redmond, M.E., and Kirby, M.L. 1989. Alteration of early vascular development after ablation of cranial neural crest. Anat. Rec. 225:209-217.

16. Leatherbury, L., Yun, J.S., and Wolfe, R. 1996. Association of abnormal configuration of the heart tube with depressed contractility after cardiac neural crest ablation. Pediatr. Res. 39:62A. (Abstr.)

17. Patten, B.M. 1922. The formation of the cardiac loop in the chick. Am.J. Anat. 30:373-397.

18. Manasek, F.J., Burnside, M.B., and Waterman, R.E. 1972. Myocardial cell shape change as a mechanism of embryonic heart looping. Dev. Biol. 29:349-371.

19. Männer, J., Seidl, W., and Steding, G. 1993. Correlation between the embryonic head flexures and cardiac development. An experimental study in chick embryos. Anat. Embryol. 188:269-285.

20. Steding, G., and Seidl, W. 1980. Contribution to the development of the heart. I. Normal development. Thorac. Cardiovasc. Surg. 28:386-409.

21. Steding, G., and Seidl, W. 1984. Cardiac septation in normal development. In Congenital heart disease: causes and processes. J.J. Nora and A. Takao, editors. Futura Publishing Co. Mt. Kisco, NY. 481-499.

22. Clark, E.B. 1986. Cardiac embryology: its relevance to congenital heart disease. Am. J. Dis. Child. 140:41-44.

23. Hamburger, V., and Hamilton, H.L. 1951. A series of normal stages in the development of the chick embryo. J. Morphol. 88:49-92.

24. Lillie, F.R. 1952. Development of the chick. H. Half \& Comp. New York, NY. 624 pp.

25. Price, R.L., Chintanowonges, C., Shiraishi, I., Borg, T.K., and Terracio, L. 1996. Local and regional variations in myofibrillar patterns in looping rat hearts. Anat. Rec. 245:83-93.

26. Brotto, M.A.D., and Creazzo, T.L. 1996. $\mathrm{Ca}^{2+}$ transients in embryonic 
chick heart: Contributions from $\mathrm{Ca}^{2+}$ channels and the sarcoplasmic reticulum. Am. J. Physiol. 270:H518-H525.

27. Creazzo, T.L., Broto, M.A., and Burch, J. 1997. Excitation-contraction coupling in the day 15 embryonic chick heart with persistent truncus arteriosus. Pediatr. Res. 42:731-737.

28. Hay, D.A., Markwald, R.R., and Fitzharris, T.P. 1984. Selected views of early heart development by scanning electron microscopy. In Scanning electron microscopy studies of embryogenesis. G.C. Schoenwolf, editor. Scanning Electron Microscopy Inc. AMF O'Hare, IL. 1983-1993.

29. Hiruma, T., and Hirakow, R. 1985. An ultrastructural topographical study on myofibrillogenesis in the heart of the chick embryo during pulsation onset period. Anat. Embryol. 172:325-329.

30. Ruzicka, D.L., and Schwartz, R.J. 1988. Sequential activation of alphaactin genes during avian cardiogenesis: vascular smooth muscle alphaactin gene transcripts mark the onset of cardiomyocyte differentiation. J. Cell Biol. 107:2575-2586.

31. Lyons, G.L. 1994. In situ analysis of the cardiac muscle gene program during embryogenesis. Trends Cardiovasc. Med. 4:70-77.

32. Wulf, E., Deboben, A., Faulstich, H., and Wieland, T.H. 1979. Fluorescent phallotoxin, a tool for the visualization of cellular actin. Proc. Natl. Acad. Sci. USA. 76:4498-4502.

33. Wunsch, A.M., Little, C.D., and Markwald, R.R. 1994. Cardiac endothelial heterogeneity defines valvular development as demonstrated by the diverse expression of JB3, an antigen of the endocardial cushion tissue. Dev. Biol. 165:585-601.

34. Tokuyasu, K.T., and Maher, P.A. 1987. Immunocytochemical studies of cardiac myofibrillogenesis in early chick embryos. I. Presence of immunofluorescent titin spots in premyofibril stages. J. Cell Biol. 105:2781-2793.

35. Kamino, K., Hirota, A., and Fujii, S. 1981. Localization of pacemaking activity in early embryonic heart monitored using voltage-sensitive dye. Nature. 290:595-597.

36. Hibbs, R.G. 1956. Electron microscopy of developing cardiac muscle in chick embryos. Am. J. Anat. 99:17-52.

37. Manasek, F.J. 1970. Histogenesis of the embryonic myocardium. Am. J. Cardiol. 25:149-168.

38. Han, Y., Dennis, J.E., Cohen-Gould, L., Bader, D.M., and Fischman, D.A. 1992. Expression of sarcomeric myosin in the presumptive myocardium of chicken embryos occurs within six hours of myocyte commitment. Dev. Dyn. 193:257-265.

39. Romanoff, A.L. 1960. The avian embryo: structural and functional development. Macmillan Co. New York, NY. 1305 pp.

40. Britsch, S., et al. 1998. The ErbB2 and ErbB3 receptors and their ligand, neuregulin-1, are essential for development of the sympathetic nervous system. Genes Dev. 12:1825-1836.

41. Moses, R.L., and Claycomb, W.C. 1982. Disorganization and reestablishment of cardiac muscle cell ultrastructure in cultured adult rat ven- tricular muscle cells. J. Ultrastruct. Res. 81:358-374.

42. Claycomb, W.C. 1983. Cardiac muscle cell proliferation and cell differentiation in vivo and in vitro. Adv. Exp. Med. Biol. 161:249-265.

43. Moses, R.L., and Claycomb, W.C. 1989. Differentiated membrane specializations and myofibrillar breakdown and recovery in cultured adult cardiac myocytes treated with TPA and diacylglycerol. J. Cell Sci. 93:95-105.

44. Bers, D.M. 1991. Excitation-contraction coupling and cardiac contractile force. Developments in cardiovascular medicine. Kluwer Academic Publishers. Dordrecht, the Netherlands. 258 pp.

45. Creazzo, T.L., Brotto, M.A.P, Lutin, W.A., and Aliff, C.L. 1995. Reduced $\mathrm{Ca}^{2+}$ currents and $\mathrm{Ca}^{2+}$ transients during early and late cardiac dysmorphogenesis. J. Physiol. (Lond.) 487:16P. (Abstr.)

46. Creazzo, T.L., Brotto, M.A.P., and Burch, J. 1995. Reduced $\mathrm{Ca}^{2+}$ currents and $\mathrm{Ca}^{2+}$ transients during early and late cardiac dysmorphogenesis. FASEB J. 9:551. (Abstr.)

47. Creazzo, T.L. 1990. Reduced "L" type calcium current in the embryonic chick heart with persistent truncus arteriosus. Circ. Res. 66:1491-1498.

48. Nosek, T.M., Fogaca, R.T.H., Hatcher, C.J., Brotto, M.A.P., and Godt, R.E. 1997. Effect of cardiac neural crest ablation on contractile force and calcium uptake and release in chick heart. Am. J. Physiol. 273:H1464-H1471.

49. Creazzo, T.L., Godt, R.E., Leatherbury, L., Conway, S.J., and Kirby, M.L. 1998. Role of cardiac neural crest cells in cardiovascular development. Annu. Rev. Physiol. 60:267-286.

50. Chen, J., Kubalak, S.W., and Chien, K.R. 1998. Ventricular muscle-restricted targeting of the RXRa gene reveals a non-cell-autonomous requirement in cardiac chamber morphogenesis. Development. 125:1943-1949.

51. Tran, C.M., and Sucov, H.M. 1998. The RXRa gene functions in a noncell-autonomous manner during mouse cardiac morphogenesis. Development. 125:1951-1956.

52. Beddington, R.S., and Robertson, E.J. 1998 Anterior patterning in mouse. Trends Genet. 14:277-284.

53. Yatskievych, T.A., Ladd, A.N., and Antin, P.B. 1997. Induction of cardiac myogenesis in avian pregastrula epiblast: the role of the hypoblast and activin. Development. 124:2561-2570.

54. Nascone, N., and Mercola, M. 1995. An inductive role for the endoderm in Xenopus cardiogenesis. Development. 121:515-523.

55. Smith, S.C., and Armstrong, J.B. 1991. Heart development in normal and cardiac-lethal mutant axolotls: a model for the control of vertebrate cardiogenesis. Differentiation. 47:129-134.

56. Sugi, Y., and Lough, J. 1994. Anterior endoderm is a specific effector of terminal cardiac myocyte differentiation of cells from the embryonic heart forming region. Dev. Dyn. 100:155-162.

57. Sater, A.K., and Jacobson, A.G. 1990. The restriction of the heart morphogenetic field in Xenopus laevis. Dev. Biol. 140:328-336.

58. Farrell, M.J., et al. 1998. HIRA, a DiGeorge syndrome candidate gene, is required for cardiac outflow tract septation. Circ. Res. 84:127-135. 